\title{
The high pressure phase transformation behavior of silicon nanowires
}

L. Q. Huston, A. Lugstein, J. S. Williams, and J. E. Bradby

Citation: Appl. Phys. Lett. 113, 123103 (2018); doi: 10.1063/1.5048033

View online: https://doi.org/10.1063/1.5048033

View Table of Contents: http://aip.scitation.org/toc/apl/113/12

Published by the American Institute of Physics

\section{Articles you may be interested in}

An optical Bragg scattering readout for nano-mechanical resonances of $\mathrm{GaN}$ nanowire arrays

Applied Physics Letters 113, 123102 (2018); 10.1063/1.5043211

Four-wave mixing in a silicon microring resonator using a self-pumping geometry

Applied Physics Letters 113, 121111 (2018); 10.1063/1.5038931

Graphene/hexagonal boron nitride heterostructures: Mechanical properties and fracture behavior from nanoindentation simulations

Applied Physics Letters 113, 121902 (2018); 10.1063/1.5047782

Spin readout via spin-to-charge conversion in bulk diamond nitrogen-vacancy ensembles

Applied Physics Letters 113, 122404 (2018); 10.1063/1.5040261

Tailoring arbitrary polarization states of light through scattering media

Applied Physics Letters 113, 121102 (2018); 10.1063/1.5048493

Branched InAs nanowire growth by droplet confinement

Applied Physics Letters 113, 123104 (2018); 10.1063/1.5045266

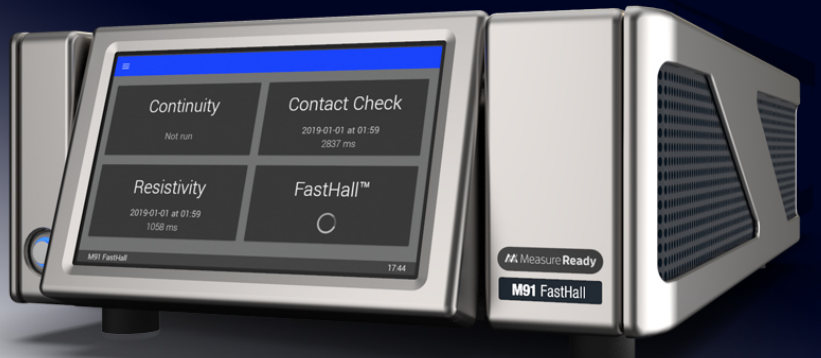

\section{Mak Measure Ready M91 FastHall ${ }^{\mathrm{TM}}$ Controller \\ A revolutionary new instrument for complete Hall analysis}




\title{
The high pressure phase transformation behavior of silicon nanowires
}

\author{
L. Q. Huston, ${ }^{1, \text { a) }}$ A. Lugstein, ${ }^{2}$ J. S. Williams, ${ }^{1}$ and J. E. Bradby ${ }^{1}$ \\ ${ }^{1}$ Research School of Physics and Engineering, The Australian National University, Acton ACT 2601, Australia \\ ${ }^{2}$ Institute for Solid State Electronics, Vienna University of Technology, Floragasse 7, A-1040 Vienna, Austria
}

(Received 10 July 2018; accepted 4 September 2018; published online 19 September 2018)

\begin{abstract}
Si nanowires of $80-150 \mathrm{~nm}$ and $200-250 \mathrm{~nm}$ diameter are pressurized up to $22 \mathrm{GPa}$ using a diamond anvil cell. Raman and x-ray diffraction data were collected during both compression and decompression. Electron microscopy images reveal that the nanowires retain a nanowire-like morphology (after high pressure treatment). On compression, dc-Si was observed to persist at pressures up to $19 \mathrm{GPa}$ compared to $\sim 11 \mathrm{GPa}$ for bulk-Si. On decompression, the metallic $\beta$-Sn phase was found to be more stable for Si nanowires compared with bulk-Si when lowering the pressure and was observed as low as $6 \mathrm{GPa}$. For the smallest nanowires studied $(80-150 \mathrm{~nm})$, predominately a-Si was obtained on decompression, whereas for larger nanowires $(200-250 \mathrm{~nm})$, clear evidence for the r8/bc8-Si phase was obtained. We suggest that the small volume of the individual Si nanowires compared with bulk-Si inhibits the nucleation of the r8-Si phase on decompression. This study shows that there is a size dependence in the high pressure behavior of Si nanowires during both compression and decompression. Published by AIP Publishing. https://doi.org/10.1063/1.5048033
\end{abstract}

Nanoscale materials are known to have electrical, mechanical, and physical properties that differ from those of their bulk counterparts. ${ }^{1}$ The phase transformation behavior of nanomaterials at high pressure is no exception. For example, some nanomaterials have been observed to require different pressures than the bulk material for a transformation to proceed. ${ }^{2,3}$ Other nanomaterials have been found to undergo different phase transformation pathways to their bulk counterparts upon compression. ${ }^{4}$ In bulk-Si, it is well established that high pressure can be used to access a number of metastable phases that have properties of technological significance such as r8-Si (rhombohedral structure, Si-XII, R $\overline{3}$ ) and bc8-Si (a body centred cubic structure, Si-III, Ia $\overline{3}){ }^{5-7}$ For example, both $\mathrm{r} 8$ and bc8-Si have been found to have interesting narrow bandgap semiconducting properties. ${ }^{5,7,8}$ Furthermore, nanowires are promising candidates for future electronic and optoelectronic devices, which can even have their properties tuned using strain, but Si nanowires (SiNWs) are only easily grown in the stable dc-Si structure. ${ }^{9,10}$ Thus, it would be interesting to explore the combination of nanowire geometry with the attractive properties of $\mathrm{r} 8-\mathrm{Si}$ and/or bc8-Si, with the aim to fabricate bc8 or r8 Si SiNWs.

To understand the high pressure behavior of SiNWs, it is first necessary to review the behavior of bulk-Si. When subjecting bulk diamond cubic (dc, Si-I, Fd $\overline{3} \mathrm{~m}$ ) Si to pressure in a diamond anvil cell (DAC), it undergoes a series of wellcharacterised phase transformations. ${ }^{11-13}$ At $\sim 11 \mathrm{GPa}$, dc-Si transforms to a metallic phase with a white tin ( $\beta$-Sn, Si-II, $\mathrm{I}_{1} /$ amd) structure. ${ }^{11,13,14}$ At higher pressures, Si will undergo a series of reversible metal to metal phase transformations such as $\beta$-Sn to Imma-Si ( $\mathrm{Si}-\mathrm{XI})$ at $13.2 \mathrm{GPa}$ and Imma to simple hexagonal (sh, Si-V, P6/mmm) at $15.6 \mathrm{GPa} .{ }^{12}$ Notably, when unloading from the $\beta$-Sn phase, Si does not revert back to dc-Si; instead, it transforms to one of a number of metastable phases. ${ }^{15} \mathrm{~A}$ fast pressure release (usually achieved in

\footnotetext{
${ }^{a)}$ Electronic mail: larissa.huston@anu.edu.au
}

nanoindentation experiments) from the $\beta$-Sn phase yields amorphous silicon (a-Si) at and below $\sim 9 \mathrm{GPa} .{ }^{16,17}$ However, slower unloading results in r8-Si at $9 \mathrm{GPa}$ which transforms to bc8-Si at $3 \mathrm{GPa} .{ }^{13}$

There have been limited studies on the phase transformation pathways of nanoscale $\mathrm{Si}$ under pressure. Studies on $10-50 \mathrm{~nm}$ Si nanoparticles and $7 \mathrm{~nm}$ diameter SiNWs suggested that $\mathrm{dc}-\mathrm{Si}$ was stable to higher pressures than bulk $\mathrm{Si}^{18,19}$ In contrast, other work reported that SiNWs around $60-80 \mathrm{~nm}$ in diameter phase transform into $\beta-\mathrm{Sn}-\mathrm{Si}$ at a lower pressure than bulk-Si, but this study did not report the behavior on decompression. ${ }^{20}$ For larger diameter SiNWs (up to $700 \mathrm{~nm}$ ), phase transformation under pressure occurred exactly like bulk $\mathrm{Si}^{21}$ In this study, the high pressure behavior of SiNWS in two size regimes $(80-150 \mathrm{~nm}$ and $200-250 \mathrm{~nm}$ diameter) was studied during compression and decompression for pressures up to $22 \mathrm{GPa}$ using Raman Spectroscopy, X-ray diffraction (XRD), scanning electron microscopy (SEM), and transmission electron microscopy (TEM).

The $80-150 \mathrm{~nm}$ SiNWs were synthesized in a hot wall CVD reactor by the VLS growth mechanism using diluted silane gas as a precursor. ${ }^{22}$ Prior to the deposition of the catalyst, the native oxide on the $\mathrm{Si}(111)$ substrates was removed with a buffered hydrofluoric acid etch to create a hydrogenterminated surface. Gold served as a catalyst, which was deposited at room temperature as a film of $2 \mathrm{~nm}$ thickness via thermal evaporation. The growth was done at a total pressure of 3 mbar using a $100 \mathrm{sccm}$ flow of silane ( $2 \%$ in a He mixture) and with $10 \mathrm{sccm}$ hydrogen at a growth temperature of $500{ }^{\circ} \mathrm{C}$. The growth was stopped by switching off the precursor gas, and the samples were cooled down in vacuum. The 200-250 nm thick Si nanowires were fabricated top down by common e-beam lithography and reactive ion etching.

The nanowires were removed from the substrate by ultrasonicating in a 4:1 mixture of methanol:ethanol or manually with a razor blade. They were then transferred into a DAC $(400 \mu \mathrm{m}$ cullet size, stainless steel gasket $\sim 50 \mu \mathrm{m}$ 
thickness, and $200 \mu \mathrm{m}$ diameter hole) using a stainless steel needle. The pressure inside the cell was determined using the ruby fluorescence method, with a small ruby ball also loaded into the DAC. ${ }^{23}$ Both Ne and 4:1 methanol:ethanol were used as a pressure medium to prevent the sample chamber from collapsing and to stop the nanowires from agglomerating together but also to create a quasihydrostatic pressure environment. The SiNWs were compressed up to $22 \mathrm{GPa}$ and monitored using insitu Raman spectroscopy with a Renishaw inVia Reflex Raman system equipped with a $532 \mathrm{~nm}$ laser and an olympus SLWD $20 \times$ objective, with a spot size of $\sim 2.6 \mu \mathrm{m}$ and an intensity of $\sim 2 \mathrm{~mW}$. XRD was conducted using $30 \mathrm{keV}$ synchrotron radiation with a Pilatus $1 \mathrm{M}-\mathrm{F}$ detector at the 16-ID-B beamline at HPCAT at the Advanced Photon Source. After unloading the SiNWs, the samples were recovered and examined using a FEI Verios SEM operating at $2 \mathrm{kV}, 13 \mathrm{pA}$, and TEM was performed with a FEI CM300 TEM operating at $300 \mathrm{kV}$.

Raman and XRD measurements for the $80-150 \mathrm{~nm}$ SiNWs up to $22 \mathrm{GPa}$ and $17 \mathrm{GPa}$ are shown in Fig. 1, respectively. The Raman measurements during compression are shown in Fig. 1(a). At $1 \mathrm{GPa}$, the Raman signal has a sharp dc-Si peak at $523 \mathrm{~cm}^{-1}$. At $14 \mathrm{GPa}$, this peak is still present but it has shifted to higher wavenumbers. It has also broadened, possibly due to the pressure medium no longer being hydrostatic. ${ }^{24}$ This peak continues to shift towards higher wavenumbers until $20 \mathrm{GPa}$ where it completely disappears, with no clear peaks from metallic phases observed above the background signal. Additionally, the second order 2TA dc-Si peak (marked with a star at $\sim 230 \mathrm{~cm}^{-1}$ ) becomes more prominent below $19 \mathrm{GPa}$. At $15 \mathrm{GPa}$, two weak peaks at $\sim 115$ and $\sim 397 \mathrm{~cm}^{-1}$ are observed. The higher wavenumber peak is observed until $18 \mathrm{GPa}$, whilst the lower wavenumber peak becomes indistinguishable from the background signal. These
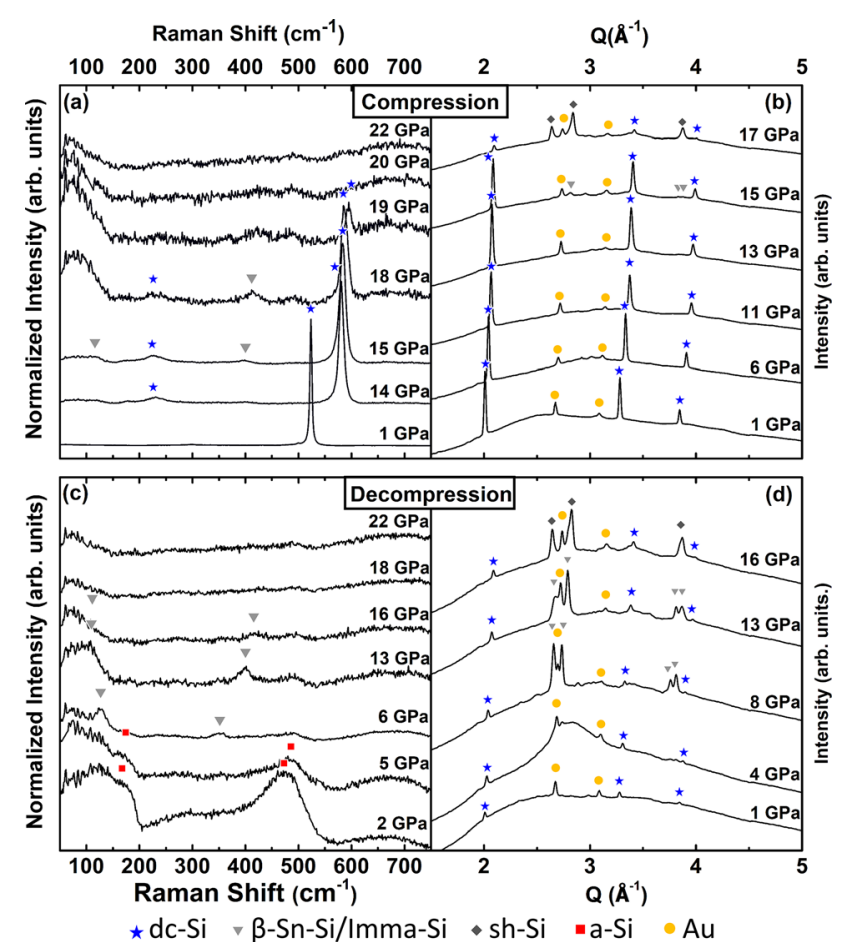

FIG. 1. In-situ Raman spectroscopy and X-ray diffraction data of 80-150 nm SiNWs taken on compression (a) and (b) and decompression (c) and (d). peaks are likely to be one of the two metallic phases, $\beta$-Sn-Si or Imma-Si, which have both been reported to exist in bulk-Si between 13.5 and $16.0 \mathrm{GPa}^{12,25}$ At $19 \mathrm{GPa}$, only a trace amount of dc-Si is observed above the background signal. The majority of the sample has likely transformed to sh-Si, which is not Raman active. ${ }^{25}$ At $20 \mathrm{GPa}$, only the background signal is observed, suggesting that the SiNWs have fully phase transformed to sh-Si.

Figure 1(b) contains a series of XRD measurements up to $17 \mathrm{GPa}$. Initially, the SiNWs are dc-Si. Note that, as well as the dc-Si peaks, there are additional peaks due to the $\mathrm{Au}$ seed used to grow the NWs. The NWs remain entirely in the dc-Si phase until $15 \mathrm{GPa}$ where a small peak is observed at $\mathrm{Q}=2.81 \AA^{-1}$ and a pair of very weak peaks are observed at $\mathrm{Q}=3.84$ and $3.88 \AA^{-1}$. These peaks correspond to either $\beta$ $\mathrm{Sn}$ or Imma-Si, but due to the low intensity of these peaks and overlap with the first Au peak, it is not possible to determine which phase is present. ${ }^{12,26}$ At the maximum pressure applied of $17 \mathrm{GPa}$, the dc-Si peaks have noticeably decreased in intensity and the $\beta$-Sn-Si or Imma-Si peaks have been replaced by strong sh-Si peaks. A small dc-Si peak indicates that not all the dc structure had transformed at this pressure, consistent with the Raman data. Thus, at $17 \mathrm{GPa}$, the SiNWs are predominately sh-Si with some traces of dc-Si.

The Raman results of the $80-150 \mathrm{~nm}$ SiNWs during decompression are shown in Fig. 1(c). At the maximum pressure of $22 \mathrm{GPa}$, the NWs were presumably completely transformed to sh-Si (not Raman active). ${ }^{25}$ At $16 \mathrm{GPa}$, two low intensity Raman modes are observed as indicated by the grey triangles. This indicates the transformation of at least some of the SiNWs to $\beta$-Sn-Si or Imma-Si. These peaks are more prominent at $13 \mathrm{GPa}$ and are observed until $6 \mathrm{GPa}$. Between $6 \mathrm{GPa}$ and $5 \mathrm{GPa}$, a final phase transformation occurs to a-Si, as indicated by the broad peaks centred at around 160 and $480 \mathrm{~cm}^{-1} \cdot{ }^{27}$

The XRD results for decompression are shown in Fig. 1(d). It is first worth noting that for all pressures, the height of the (dc-Si) peaks from the remaining untransformed dc-Si (at $17 \mathrm{GPa}$ ) did not change significantly. This suggests that no additional dc-Si is formed during decompression. However, there were changes observed in the metallic phases. Between $16 \mathrm{GPa}$ and $13 \mathrm{GPa}$, the sh-Si phase transforms into $\beta$-Sn-Si (most likely via the Imma-Si phase) which continued to exist until at least $8 \mathrm{GPa}$ and disappears entirely between $8 \mathrm{GPa}$ and $4 \mathrm{GPa}$. This disappearance is most likely due to the amorphisation of the NWs, as observed in the Raman spectra. It should be noted that the XRD signal of a-Si has very broad features and is very weak, given that the nanowires are loosely packed in the DAC. Also, in both experiments shown in Fig. 1, the SiNWs were decompressed in relatively large pressure increments. Given that it is known from nanoindentation experiments in $\mathrm{Si}$ that a fast decompression rate can favor the formation of a-Si over the crystalline metastable phases (bc8-Si and r8-Si), a new batch of SiNW was compressed to $20 \mathrm{GPa}$ and decompressed from $\sim 8 \mathrm{GPa}$ very slowly in $\sim 0.2 \mathrm{GPa}$ increments over a day. ${ }^{17}$ It was found (using Raman Spectroscopy) that the SiNWs were still predominately a-Si but a trace amount of bc8-Si was also observed. 
The Raman spectra of the 200-250 nm SiNWs in compression and decompression are shown in Figs. 2(a) and 2(b), respectively. On compression, dc-Si was found to start transforming to $\beta$-Sn-Si/Imma-Si at $15 \mathrm{GPa}$ as seen by the presence of two weak peaks indicated by grey triangles. At $20 \mathrm{GPa}$, these peaks have disappeared, again indicating a transition to sh-Si. On decompression, $\beta$-Sn-Si is found to form at 16 GP (possibly with some Imma-Si present), and the SiNWs remain entirely in a metallic phase (presumably $\beta-\mathrm{Sn}-\mathrm{Si}^{11}$ ) $7.9 \mathrm{GPa}$. At this pressure, additional peaks (indicated by green crosses) are observed, corresponding to r8-Si. ${ }^{28}$ Some $\beta$-Sn-Si is still present at this pressure, and it remains until between $5.8 \mathrm{GPa}$ and $5.1 \mathrm{GPa}$ where it appears to be replaced by a-Si (red squares) as indicated by the presence of two broad peaks.

To determine the morphology and structural integrity of the SiNWs after compression, scanning electron microscopy (SEM) was performed. Figures 3(a) and 3(b) show SEM and TEM images of the SiNWs before compression, respectively, and Fig. 3(c) shows an SEM image of a gasket full of 80-150 nm SiNWs after being removed from a DAC after compression to $17 \mathrm{GPa}$. The inset shows a region at a higher magnification. From these images, it is quite clear that the SiNWs maintained the nanowire shape during compression and did not merge together to form a bulk-like structure. Transmission electron microscopy was also performed on individual SiNWs. A bright field image with an inset of the selected area diffraction pattern (SADP) of an $80-150 \mathrm{~nm}$ SiNW loaded up to $22 \mathrm{GPa}$ is shown in Fig. 3(d). The SADP shows a number of diffuse rings which correspond to a-Si, consistent with the XRD and Raman data. This image shows that the SiNW returned to essentially the same geometry as before it was loaded.

The high pressure behavior of SiNWs from this study and that of bulk-Si from the literature (Refs. 11 and 29) is summarized in Fig. 4. This figure shows the phases present in bulk-Si, 200-250 nm SiNWs, and 80-150 nm SiNWs at the pressures shown on the bottom axis. Compression is shown on the left and decompression is shown on the right. Solid colors represent a single phase being present whilst vertical lines indicate a transition region where two phases

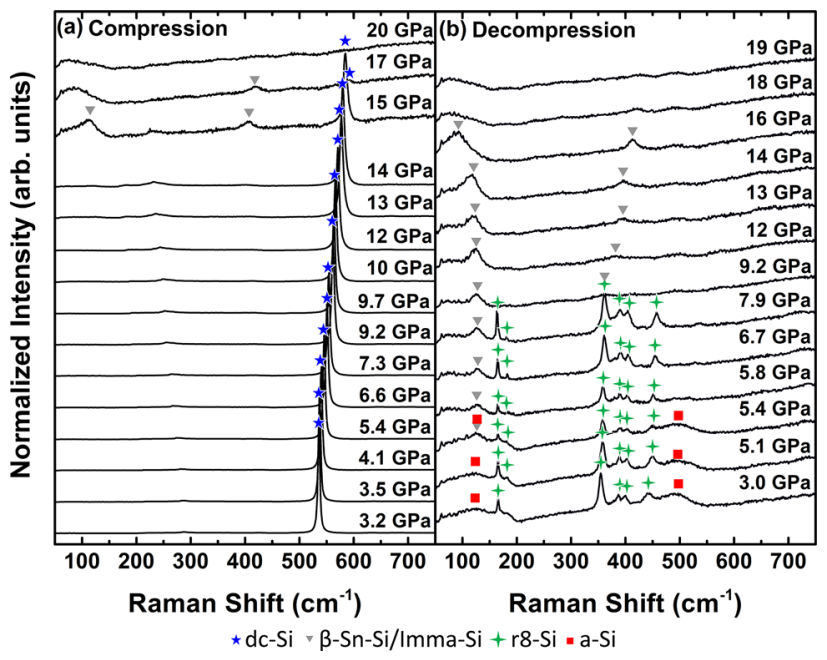

FIG. 2. Raman spectra of 200-250 nm SiNWs taken on (a) compression and (b) decompression.

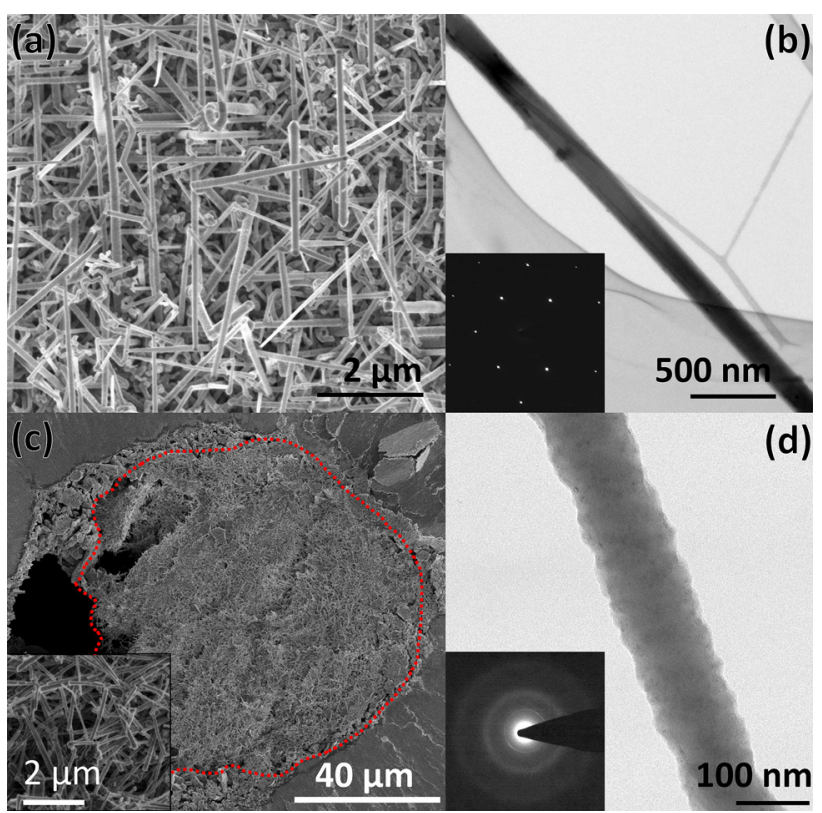

FIG. 3. (a) SEM image of SiNWs before compression. (b) Bright-field TEM image of a SiNW before compression selected area diffraction pattern (SADP) inset taken from the SiNW shown. (c) SEM image of 80-150 nm SiNWs after compression to $17 \mathrm{GPa}$. The SiNWs are within the red circled region and the outer region is the gasket. The inset is a higher magnification image. (d) Bright-field TEM image of a SiNW compressed to $22 \mathrm{GPa}$ with a SADP inset taken from the SiNW shown.

are present. On compression, the onset and completion of phase transformation to metallic phases for both diameters were found to be much higher than in bulk-Si. This is consistent with a study of $10-50 \mathrm{~nm} \mathrm{Si} \mathrm{nanoparticles}{ }^{18}$ and a further study where no phase transformation was reported up to 15.6 GPa. ${ }^{19}$ In contrast, two other studies found SiNWs to phase transform to metallic phases at similar pressure to bulk-Si. ${ }^{20,21}$ In the first of these studies, a large size distribution of 50-700 nm SiNWs was used, suggesting that the large SiNWs may have biased the behavior to that of bulk-Si. ${ }^{21}$ The other study found the onset of phase transformation to be slightly lower than bulk-Si ${ }^{20}$ but this observation could be a result of the manner in which the SiNWs were loaded into the DAC causing an agglomeration of SiNWs and hence bulk-like behavior.

On decompression, the SiNWs in this current study were found to transform from sh-Si to $\beta$-Sn-Si/Imma-Si at a

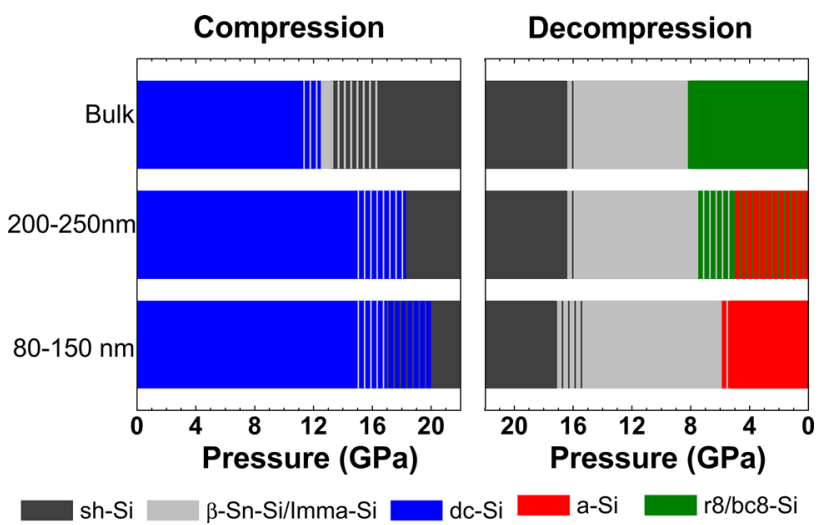

FIG. 4. A summary of the high pressure phase transformation behavior of $\mathrm{Si}$ at different sizes. Bulk-Si data are from Refs. 11, 12, and 29. 
similar pressure to bulk-Si. However, $\beta$-Sn-Si was found to be present at pressures as low as $\sim 5 \mathrm{GPa}$ in the SiNWs, consistent with previous observations for $10-50 \mathrm{~nm}$ Si nanoparticles. $^{18}$ For the larger 200-250 nm SiNWs, partial transformation to r8-Si was observed at a slightly lower pressure than bulk-Si. ${ }^{29}$ The remaining $\beta$-Sn-Si appeared to transform to a-Si at $\sim 5 \mathrm{GPa}$, as revealed by a significant additional a-Si phase present, similar to the $80-150 \mathrm{~nm}$ SiNWs. We therefore deduce from our work (and the few previous studies) that the phase transformation behavior of $\mathrm{Si}$ depends strongly on the particle/SiNW size. Isolated SiNWs of a larger size (approximately 600-700 $\mathrm{nm}+$ ) exhibit bulk-like phase transformation behavior. At smaller sizes, Si particles/SiNWs exhibit elevated pressures for the dc-Si to metallic phase transformation upon compression and depressed phase transformation pressures during decompression, with a-Si being the dominant end phase for the smallest sizes (below $200 \mathrm{~nm}$ ) and $\mathrm{r} 8 / \mathrm{bc} 8$-Si occurring for intermediate sizes.

We suggest that the suppression of the dc-Si to metallic phase transition is a direct result of difficulty in nucleating the $\beta$-Sn phase. It should be noted that, even in bulk-Si, $\beta$ $\mathrm{Sn}-\mathrm{Si}$ is difficult to nucleate, being described as "sluggish" in the literature. ${ }^{30}$ This phase transformation is considered as a reconstructive transition since it involves the breaking of dc-Si bonds to form $\beta$-Sn-Si, thus requiring a significant amount of energy. ${ }^{31}$ We propose that this phase is even more difficult to nucleate in the SiNWs, as a result of both the higher surface to volume ratio and the smaller volume of individual SiNWs compared with bulk-Si. Since $\beta$-Sn-Si is already difficult to nucleate in bulk-Si, fewer potential nucleation sites that are available in a SiNW may raise the pressure necessary to initiate phase transformation to $\beta$-Sn/Imma for SiNWs. For smaller SiNWs, the data suggest that there is a direct dc-Si to sh-Si phase transformation at and above $17 \mathrm{GPa}$. For the $200-250 \mathrm{~nm}$ SiNWs, the situation is not so clear cut and a dc-Si to $\beta$-Sn/Imma-Si may be occurring at $15 \mathrm{GPa}$ prior to sh-Si formation at higher pressures.

It was mentioned earlier that a sh-Si to $\beta$-Sn-Si/Imma-Si phase transformation on decompression occurs at similar pressures for all sizes of nanoparticles/NWs. This metallic to metallic phase transition is displacive, involving the movement of some atoms in the unit cell from the sh-Si positions to those in the Imma and $\beta$-Sn phases. ${ }^{31}$ A theoretical study of high pressure phases of Si found that the total free energies $\beta$-Sn-Si, Imma-Si, and sh-Si are similar. ${ }^{32}$ These similarities in total energy and the displacive nature of the atomic transitions may explain why there is little to no size effect on the sh-Si to Imma-Si/ $\beta$-Sn-Si (metallic to metallic) phase transformation of SiNWs compared with bulk-Si.

As decompression continues, the metallic nature of the bonding changes to covalent bonding with the $\beta$-Sn-Si to r8$\mathrm{Si}$ phase transformation. Following on from the previous argument, it would be expected that there is a significantly high kinetic barrier to nucleation of $\mathrm{r} 8$-Si. As the small size (volume) of SiNWs limits the number of potential nucleation sites for $\mathrm{r} 8-\mathrm{Si}$, the SiNWs may remain as $\beta-\mathrm{Sn}-\mathrm{Si}$ until significantly lower pressures compared to bulk-Si. Hence, we might expect that there would exist an increased difficulty in nucleating $\mathrm{r} 8$-Si from $\beta$-Si-Si on decompression compared with bulk-Si. We further suggest that the formation of a-Si upon decompression of SiNWs may be similar to rapid unloading during nanoindentation experiments. ${ }^{16,17}$ In nanoindentation, a sharp diamond tip pressed into $\mathrm{Si}$ forms $\beta$-Sn$\mathrm{Si}$ and rapid decompression leads to the formation of a-Si when there is insufficient time to nucleate $\mathrm{r} 8$ - $\mathrm{Si}$ within the small $\beta$-Sn volume. ${ }^{17}$ However, by slowly unloading the indenter tip, r8/bc8-Si can be formed. ${ }^{17}$ In terms of our observed behavior of SiNWs during decompression, we suggest that for the 200-250 nm SiNWs, the smaller volume (like the nanoindentation case) results in a nucleation problem in $\mathrm{r} 8$-Si formation. Incomplete transformation to $\mathrm{r} 8 \mathrm{-Si}$ occurs by $5 \mathrm{GPa}$ and the remaining $\beta$-Sn-Si then transforms to a-Si somewhat like fast unloading for nanoindentation. In smaller SiNWs (80-150 nm), there appears to be almost total suppression of r8-Si nucleation and a-Si forms directly from $\beta$-Sn-Si at and below $6 \mathrm{GPa}$. In light of these observations, it would be interesting to explore the temperature dependence of phase transformation of SiNWs during decompression, since it has previously been possible in nanoindentation studies to enhance the formation of $\mathrm{r} 8 / \mathrm{bc} 8$-Si phases by increasing the temperature. ${ }^{33}$

In conclusion, a clear size effect in some but not all of the pressure-induced phase transformations of SiNWs was observed during both compression and decompression. On compression, dc-SiNWs are found to suppress phase transformation to metallic phases until higher pressures than for bulk-Si, possibly even a direct dc to sh phase transformation for smaller SiNWs at and above $17 \mathrm{GPa}$. During decompression, the sh-Si to Imma/ $\beta$-Sn-Si transformation was found to be similar to bulk Si. However, $\beta$-Sn-Si was found to remain until much lower pressures than bulk-Si. For the intermediate size SiNWs, strong evidence was observed for a phase transformation to r8-Si that occurred at pressures between $\sim 8$ and $5 \mathrm{GPa}$ on decompression but significant a-Si occurred below $\sim 5 \mathrm{GPa}$. For the smallest SiNWs below $200 \mathrm{~nm}$, a phase transformation to predominately a-Si occurred below $6 \mathrm{GPa}$.

L.Q.H. acknowledges her support from an Australian Government Research Training Program Scholarship. J.E.B. would like to acknowledge funding from the ARC Future Fellowship Scheme. A.L. acknowledges financial support from the Austrian Science Fund (FWF): Project No. P28175N27 and e-beam lithography support by Manfred Reiche from the Max Planck Institute of Microstructure Physics, Halle, Germany. This work was performed in part at the ACT node of the Australian National Fabrication Facility, a company established under the National Collaborative Research Infrastructure Strategy to provide nano- and microfabrication facilities for Australian researchers. We also acknowledge the ACT node of the Australian Microscopy and Microanalysis Research Facility for use of the TEM facilities. We would like to thank Thomas Shiell for his assistance with the XRD measurements and Lachlan Smillie and Jennifer Wong-Leung for their help with the measurement of the initial SiNWs via TEM and preliminary TEM measurements. We would like to acknowledge the use of HPCAT facilities. HPCAT operations were supported by DOE-NNSA under Award No. DE-NA0001974 and DOEBES under Award No. DE-FG02-99ER45775, with partial instrumentation funding by NSF. APS was supported by 
DOE-BES, under Contract No. DE-AC02-06CH11357. We would like to thank Sergey Tkachev for his assistance with gas loading. The use of the COMPRES-GSECARS gas loading system was supported by COMPRES under NSF Cooperative Agreement No. EAR-1606856 and by GSECARS through NSF Grant No. EAR-1634415 and DOE Grant No. DE-FG02-94ER14466. This research used resources of the Advanced Photon Source, a U.S. Department of Energy (DOE) Office of Science User Facility operated for the DOE Office of Science by Argonne National Laboratory under Contract No. DE-AC02-06CH11357.

${ }^{1}$ Springer Handbook of Nanomaterials, edited by R. Vajtai (SpringerVerlag, Berlin, Heidelberg, London, New York, 2013).

${ }^{2}$ S. H. Tolbert and A. P. Alivisatos, J. Chem. Phys. 102, 4642 (1995).

${ }^{3}$ Q. Guo, Y. Zhao, W. L. Mao, Z. Wang, Y. Xiong, and Y. Xia, Nano Lett. 8, 972 (2008)

${ }^{4}$ L. Wang, W. Yang, Y. Ding, Y. Ren, S. Xiao, B. Liu, S. V. Sinogeikin, Y. Meng, D. J. Gosztola, G. Shen et al., Phys. Rev. Lett. 105, 095701 (2010). ${ }^{5}$ B. D. Malone, J. D. Sau, and M. L. Cohen, Phys. Rev. B 78, 161202(R) (2008).

${ }^{6}$ H. Zhang, H. Liu, K. Wei, O. O. Kurakevych, Y. Le Godec, Z. Liu, J. Martin, M. Guerrette, G. S. Nolas, and T. A. Strobel, Phys. Rev. Lett. 118, 146601 (2017).

${ }^{7}$ J. D. Joannopoulos and M. L. Cohen, Phys. Rev. B 7, 2644 (1973).

${ }^{8}$ S. Ruffell, K. Sears, J. E. Bradby, and J. S. Williams, Appl. Phys. Lett. 98, 052105 (2011).

${ }^{9}$ W. Lu and C. M. Lieber, J. Phys. D: Appl. Phys. 39, R387 (2006).

${ }^{10}$ A. Lugstein, M. Steinmair, A. Steiger, H. Kosina, and E. Bertagnolli, Nano Lett. 10, 3204 (2010).

${ }^{11}$ J. Z. Hu, L. D. Merkle, C. S. Menoni, and I. L. Spain, Phys. Rev. B 34, 4679 (1986).
${ }^{12}$ M. I. McMahon, R. J. Nelmes, R. I. Wright, and D. R. Allan, Phys. Rev. B 50, 739 (1994).

${ }^{13}$ R. O. Piltz, J. R. Maclean, S. J. Clark, G. J. Ackland, P. D. Hatton, and J. Crain, Phys. Rev. B 52, 4072 (1995).

${ }^{14}$ J. C. Jamieson, Science 139, 762 (1963).

${ }^{15}$ J. S. Kasper and S. M. Richards, Acta Cryst. 17, 752 (1964).

${ }^{16}$ V. Domnich, Y. Gogotsi, and S. Dub, Appl. Phys. Lett. 76, 2214 (2000).

${ }^{17}$ J. E. Bradby, J. S. Williams, J. Wong-Leung, M. V. Swain, and P. Munroe, J. Mater. Res. 16, 1500 (2001).

${ }^{18}$ S. H. Tolbert, A. B. Herhold, L. E. Brus, and A. P. Alivisatos, Phys. Rev. Lett. 76, 4384 (1996).

${ }^{19}$ S. Khachadorian, K. Papagelis, H. Scheel, A. Colli, A. C. Ferrari, and C. Thomsen, Nanotechnology 22, 195707 (2011), ISSN 0957-4484.

${ }^{20}$ Y. Wang, J. Zhang, J. Wu, J. L. Coffer, Z. Lin, S. V. Sinogeikin, W. Yang, and Y. Zhao, Nano Lett. 8, 2891 (2008), pMID: 18720974.

${ }^{21}$ H. K. Poswal, N. Garg, S. M. Sharma, E. Busetto, S. K. Sikka, G. Gundiah, F. L. Deepak, and C. N. R. Rao, J. Nanosci. Nanotechnol. 5, 729 (2005), ISSN 1533-4880.

${ }^{22}$ R. S. Wagner and W. C. Ellis, Appl. Phys. Lett. 4, 89 (1964).

${ }^{23}$ A. Dewaele, M. Torrent, P. Loubeyre, and M. Mezouar, Phys. Rev. B 78, 104102 (2008).

${ }^{24}$ J. D. Barnett, S. Block, and G. J. Piermarini, Rev. Sci. Instrum. 44, 1 (1973).

${ }^{25}$ H. Olijnyk, Phys. Rev. Lett. 68, 2232 (1992).

${ }^{26}$ M. I. McMahon and R. J. Nelmes, Phys. Rev. B 47, 8337 (1993).

${ }^{27}$ T. Ishidate, K. Inoue, K. Tsuji, and S. Minomura, Solid State Commun. 42, 197 (1982).

${ }^{28}$ H. Olijnyk and A. P. Jephcoat, Phys. Status Solidi B 211, 413 (1999).

${ }^{29}$ J. Crain, G. J. Ackland, J. R. Maclean, R. O. Piltz, P. D. Hatton, and G. S. Pawley, Phys. Rev. B 50, 13043 (1994).

${ }^{30}$ R. H. Wentorf, Jr. and J. S. Kasper, Science 139, 338 (1963).

${ }^{31}$ A. Mujica, A. Rubio, A. Munõz, and R. J. Needs, Rev. Mod. Phys. 75, 863 (2003).

${ }^{32}$ S. P. Lewis and M. L. Cohen, Phys. Rev. B 48, 16144 (1993).

${ }^{33}$ M. Kiran, T. Tran, L. Smillie, B. Haberl, D. Subianto, J. Williams, and J. Bradby, J. Appl. Phys. 117, 205901 (2015). 